\title{
On the relevance of analytical film thickness EHD equations for isothermal point contacts: Qualitative or quantitative predictions?
}

\author{
Jean-David WHEELER, Philippe VERGNE* , Nicolas FILLOT, David PHILIPPON \\ Univ Lyon, INSA Lyon, CNRS, LaMCoS-UMR5259, Villeurbanne F-69621, France \\ Received: 04 November 2016 / Revised: 19 November 2016 / Accepted: 20 November 2016 \\ (C) The author(s) 2016. This article is published with open access at Springerlink.com
}

\begin{abstract}
Thin film and elastohydrodynamic lubrication regimes are rather young domains of tribology and they are still facing unresolved issues. As they rely upon a full separation of the moving surfaces by a thin (or very thin) fluid film, the knowledge of its thickness is of paramount importance, as for instance to developing lubricated mechanisms with long lasting and efficient designs. As a consequence, a large collection of formulae for point contacts have been proposed in the last 40 years. However, their accuracy and validity have rarely been investigated. The purpose of this paper is to offer an evaluation of the most widespread analytical formulae and to define whether they can be used as qualitative or quantitative predictions. The methodology is based on comparisons with a numerical model for two configurations, circular and elliptical, considering both central and minimum film thicknesses.
\end{abstract}

Keywords: thin film lubrication; elastohydrodynamic lubrication (EHL); film thickness prediction; EHD analytical equation; central film thickness; minimum film thickness; circular contacts; elliptical contacts

\section{Introduction}

For almost four decades, semi-analytical expressions (simply named analytical in the following) were proposed to calculate film thickness in elastohydrodynamic lubrication (EHL) and especially for point contacts. They generally aimed to predict central and minimum film thicknesses $\left(h_{\mathrm{c}}\right.$ and $\left.h_{\mathrm{m}}\right)$ in elastohydrodynamic (EHD) circular contacts under pure rolling and isothermal conditions, and for lubricants considered as Newtonian fluids. Numerous formulas have been published, in particular during the last two decades during which progress in both experimental and computational techniques was substantial. They have been widely used by researchers to advance the knowledge in the fields of thin film lubrication and EHL, and by design and development engineers for estimating film thickness in mechanical devices, like gearboxes, rolling element bearings, cam-tappet assemblies, piston-ring-

* Corresponding author: Philippe VERGNE.

E-mail: philippe.vergne@insa-lyon.fr liner systems, etc.

Surprisingly, the accuracy of the existing film thickness relationships has rarely been investigated in detail, and their application within the conditions for which they were originally established was not often verified nor respected. To the best of the authors' knowledge, very few-if not any-papers have dealt with these concerns. Except maybe those of van Leeuwen $[1,2]$ of whom it was not the primary objective: his aim was to derive the most accurate values of viscositypressure coefficients from, on one side, central film thickness measurements performed in circular contacts and, on the other side, a wide collection of EHD film thickness equations. Though indirectly, he showed that certain expressions were more relevant than others through their ability to provide correct values of viscosity-pressure coefficients. This is, however, a typical illustration of the classical approach of EHL, in which the author has chosen to derive the lubricants' properties from film thickness or friction measurements instead of relying on direct rheological measurements, obtained independently of tribological tests. 


\begin{tabular}{|c|c|c|c|}
\hline \multicolumn{4}{|c|}{ Nomenclature } \\
\hline$a$ & $\begin{array}{l}\text { contact length or dimension in the } \\
\text { entrainment direction }(\mathrm{m})\end{array}$ & $R_{y}$ & $\begin{array}{l}\text { reduced radius of curvature perpendicular } \\
\text { to the entrainment direction }(\mathrm{m})\end{array}$ \\
\hline$b$ & contact width or dimension perpendicular & $T_{0}$ & inlet temperature $(\mathrm{K})$ \\
\hline & to the entrainment direction $(\mathrm{m})$ & $u_{\mathrm{e}}$ & mean entrainment velocity \\
\hline$D$ & ratio of reduced radii of curvature, & & $(\mathrm{m} / \mathrm{s})=\left(u_{1}+u_{2}\right) / 2$ \\
\hline & $D=R_{x} / R_{y}$ & $u_{1}, u_{2}$ & velocity in the $x$-direction of surfaces \\
\hline$E_{1}, E_{2}$ & Young modulii of solids 1 and $2(\mathrm{~Pa})$ & & 1 and $2(\mathrm{~m} / \mathrm{s})$ \\
\hline$E^{\prime}$ & $\begin{array}{l}\text { reduced modulus of elasticity }(\mathrm{Pa}) \\
2 / E^{\prime}=\left(1-v_{1}^{2}\right) / E_{1}+\left(1-v_{2}^{2}\right) / E_{2}\end{array}$ & $U$ & $\begin{array}{l}\text { dimensionless speed parameter } \\
\text { (Hamrock \& Dowson })=\mu \cdot u_{e} /\left(E^{\prime} \cdot R_{x}\right)\end{array}$ \\
\hline G & dimensionless material parameter & $w$ & normal load $(\mathrm{N})$ \\
\hline & $($ Hamrock \& Dowson $)=\alpha^{*} \cdot E^{\prime}$ & $W$ & dimensionless load parameter \\
\hline$h_{\mathrm{c}}$ & central film thickness (m) & & $($ Hamrock \& Dowson $)=w /\left(E^{\prime} \cdot R_{x}^{2}\right)$ \\
\hline$h_{\mathrm{m}}$ & minimum film thickness (m) & $\alpha^{*}$ & reciprocal asymptotic isoviscous pressure, \\
\hline$k$ & ellipticity ratio $=\frac{b}{a}$ & & according to Blok [21] $\left(\mathrm{Pa}^{-1}\right)$ \\
\hline$L$ & dimensionless material parameter & $\mu$ & lubricant dynamic viscosity $(\mathrm{Pa} \cdot \mathrm{s})$ \\
\hline & $($ Moes $)=G \cdot(2 U)^{0.25}$ & $\mu_{0}$ & lubricant dynamic viscosity (Pa.s) at the \\
\hline$M$ & dimensionless load parameter (Moes) for & & inlet temperature $T_{0}$ \\
\hline & point contact $=W /(2 U)^{0.75}$ & $\rho_{0}$ & lubricant density $\left(\mathrm{kg} \cdot \mathrm{m}^{-3}\right)$ at the inlet \\
\hline$p_{\mathrm{H}}$ & Hertzian pressure (MPa) & & temperature $T_{0}$ \\
\hline$R_{x}$ & $\begin{array}{l}\text { reduced radius of curvature in the } \\
\text { entrainment direction }(\mathrm{m})\end{array}$ & $\sigma$ & $\begin{array}{l}\text { composite roughness of the mating } \\
\text { surfaces }(\mathrm{m})\end{array}$ \\
\hline
\end{tabular}

Given the current trends towards more and more severe conditions applied to lubricated mechanisms due to technological, economic, and environmental constraints, and the unceasing film thickness decrease in lubricated contacts, the need to predict film thickness with high precision appears more than ever well founded. Specifically, new important questions have emerged and require clarification and verification, as for instance:

(1) a deviation of 10 or $20 \mathrm{~nm}$, which seemed negligible 40 years ago, can nowadays have some dramatic consequences on the integrity of the mechanisms: this justifies the assessment of the analytical equations currently in use to make sure they are accurate enough;

(2) the relevance of the extrapolation to often much lower thicknesses as those used to design the analytical expressions should be checked to consider the latter appropriate for predicting very thin film thicknesses.

Furthermore, the related literature generally deals with central film thickness, $h_{c}$ whereas it is well known that $h_{\mathrm{m}}$, the minimum film thickness, is the crucial parameter for determining the lubrication regime through the $h_{\mathrm{m}} / \sigma$ ratio, $\sigma$ being the composite roughness of the mating surfaces. Finally, in many applications the actual geometry of the contacting bodies leads to elliptical point contacts. These latter can be narrow (i.e., slender configuration) or wide, according to the orientation of the larger equivalent radius of curvature of the mating bodies with respect to the main rolling direction. Elliptical point contacts have received much less attention compared to circular ones and, as a consequence, a limited number of analytical expressions were published for the former.

Therefore, the aim of this work is to provide a new insight into the validity and accuracy of some among the most widely used analytical film thickness equations, established for circular and elliptical contacts. From a set of operating parameters leading to 5 reference cases, they will be confronted to a full EHD numerical model, taken here as a reference due to the numerous conditions considered for achieving its validation against 
experimentation. Both the central and minimum film thicknesses will be studied in the case of a Newtonian lubricant operated under pure rolling and isothermal conditions. The purpose of this comparison is indeed not to rank the models against each other, but to evidence whether they can be considered sufficiently quantitative or just qualitative, in the domains investigated in this work.

\section{Models and conditions}

The choice of a reasonable number of EHD film thickness equations to be included in this work was dictated by different criteria (extensive use, circular and/or elliptical geometry). The widely-used expressions mentioned below were selected on the basis of (i) van Leeuwen studies [1,2] and (ii) a previous experimental work [3] in which the capabilities of some of them were quantitatively compared with measurements performed over wide ranges of operating conditions and for numerous lubricants of different nature:

(1) Hamrock \& Dowson [4], for circular and elliptical (wide only) contacts;

(2) Nijenbanning et al. [5] for $h_{c}$ in circular and elliptical (wide only) contacts, combined with Chevalier $h_{\mathrm{c}} / h_{\mathrm{m}}$ table [6] for calculating $h_{\mathrm{m}}$ (see Ref. [3]) in circular contacts;

(3) Evans \& Snidle [7], for circular contacts only;

(4) Chittenden et al. [8], for circular and elliptical (slender and wide) contacts;

(5) Masjedi \& Khonsari [9], for circular and elliptical (wide only) contacts.

The analytical expressions and the numerical tables corresponding to these EHD film thickness equations and $h_{\mathrm{c}} / h_{\mathrm{m}}$ ratios are given in Appendix.

Figure 1 provides a schematic description of the domains on which the analytical models above were established, as a function of $M$ and $L$, the dimensionless load and material parameters as proposed originally by Moes [10] ( $M$ and $L$ are defined in the Nomenclature). These ranges take into account the indirect $(M, L)$ variations produced when considering elliptical contacts, except in the case of the Evans \& Snidle equation which concerns circular cases only. Overall, the domains of validity of the analytical models, expressed in a $(M, L)$ chart in Fig. 1, cover well the full range of EHL. However some of them were restricted

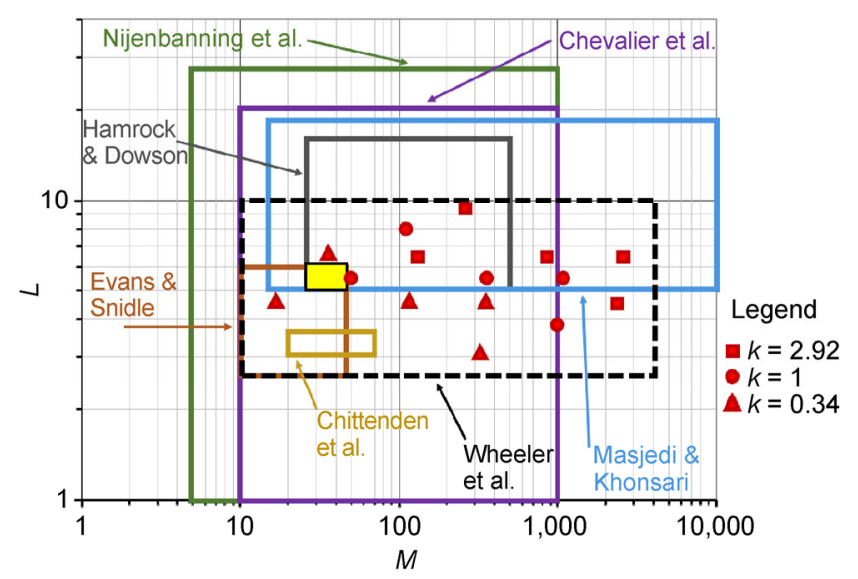

Fig. 1 Domains (expressed by empty rectangles) on which EHD film thickness equations for circular and elliptical contacts were established. The yellowed area represents the common area covered by all the analytical expressions considered here. The black bold dotted line indicates the region where the full numerical model was applied in Ref. [11]. The symbols show the domain corresponding to the 5 references cases of Table 1 , in the circular $(k=1)$ and the elliptical configurations ( $k=2.92$ or 0.34 ).

to rather limited $(M, L)$ areas and extrapolation could result in inaccurate results. There is a common area covered by all analytical models, given that Chittenden et al. [8] have also incorporated the results of Hamrock \& Dowson [4] to derive their equations. This overlap extends to values of $M$ and $L$ between 25 and 45, and between 5 and 6 , respectively, see the yellowed rectangle in Fig. 1. This area ultimately represents a very narrow domain compared to the full field of EHL.

The versatile EHD model used here as a reference has been already presented in Ref. [11] and will not be detailed further. It results from recent modeling developments performed at LaMCoS, after the works of Doki-Thonon in the case of spinning EHD contacts $[12,13]$ and those of Habchi who has laid the foundations inherent to this multiphysics model $[14,15]$. The steady state problem concerns smooth surfaces, fully flooded, Newtonian and isothermal conditions. Based on the finite element method, the numerical model solves simultaneously the Reynolds, the solids deformation and the load balance equations. Typically, the Reynolds equation was solved using $2 \times 10^{4}$ degrees of freedom and the convergence was achieved when a relative deviation lower than $10^{-3}$ was obtained.

The physical behavior of the lubricant is taken into account through (i) a rheological equation which describes the viscosity changes with pressure and (ii) 
a classical equation of state for the density variations: the Newtonian viscosity follows a modified WilliamsLandel-Ferry (WLF) correlation [16] and the density varies according to the Murnaghan [17] equation. Both constitutive equations were fitted to independent characterizations carried out with high pressure devices, see Ref. [11] for more details.

Since its early developments, quantitative comparisons with experiment have proven the reliability and accuracy of this numerical model to predict film thickness in various configurations: for instance with conventional (mineral turbine oil [14]) and nonconventional lubricants (low viscosity working fluids or glycerol $[18,19])$, or under complex kinematic conditions (spinning-skewing EHD contacts [13]). More recently, it was adapted for non-circular EHD contacts and successfully validated by quantitative confrontation with experiments [20].

A first reference case (called Case 3) was defined in the circular configuration, with $R_{\mathrm{x}}=R_{\mathrm{y}}=80 \mathrm{~nm}$, an entrainment velocity $u_{\mathrm{e}}=2 \mathrm{~m} / \mathrm{s}$, a normal load $w=$ $800 \mathrm{~N}$, a bearing steel material for the two solids $(E=$ $210 \mathrm{GPa}, v=0.3)$, and an inlet lubricant temperature $T_{0}=313 \mathrm{~K}$, giving $\alpha^{*}=20 \mathrm{GPa}^{-1}, \mu_{0}=0.008 \mathrm{~Pa} \cdot \mathrm{s}$ and $\rho_{0}=863 \mathrm{~kg} \cdot \mathrm{m}^{-3}$. Then both the entrainment velocity, $u_{\mathrm{e}}$, and the normal load, $w$, were varied in order to define 4 other reference cases to cover sufficient wide ranges of operating conditions, see Table 1 where they are also reported and expressed by the $(M, L)$ dimensionless parameters. Apart from the central Case 3 already described, a low $(120 \mathrm{~N})$ and a high $(2,500 \mathrm{~N})$ normal load condition together with a low $(0.5 \mathrm{~m} / \mathrm{s})$ and a high $(10 \mathrm{~m} / \mathrm{s})$ entrainment speed condition are proposed. From these physical values, it is possible to compute the corresponding dimensionless

Table 1 Normal load, entrainment velocity (both in bold) and Hertzian contact pressure for the circular configuration (in italic) of the five reference cases. The $(M, L)$ values are given under the Case number.

\begin{tabular}{ccccc}
\hline & & \multicolumn{4}{c}{$\boldsymbol{u}_{\mathrm{e}}(\mathbf{m} / \mathbf{s})$} \\
\cline { 2 - 5 } & & $\mathbf{0 . 5}$ & $\mathbf{2}$ & $\mathbf{1 0}$ \\
\hline & $\mathbf{1 2 0}$ & - & Case 1 & - \\
& 364 & & $(51,5.2)$ & \\
\cline { 2 - 5 } $\boldsymbol{w}(\mathbf{N})$ & $\mathbf{8 0 0}$ & Case 2 & Case 3 & Case 4 \\
$P_{\mathrm{h}}(\mathrm{MPa})$ & 686 & $(962,3.7)$ & $(340,5.2)$ & $(102,7.8)$ \\
& $\mathbf{2 5 0 0}$ & - & Case 5 & - \\
& 1002 & - & $(1,062,5.2)$ & - \\
\hline
\end{tabular}

parameters $M$ and $L$, and to compare them with those of Fig. 1 for the analytical models.

In the circular configuration, the 5 reference cases of Table 1 lead to a domain defined by $M \in[50,1,062]$ and $L \in[3.7,7.8]$, see the red dots in Fig. 1. Moreover in Ref. [11], the numerical experimentations covered a larger range delimited by $M \in[10,4,000]$ and $L \in$ $[2.5,10]$, highlighted by the black dotted contour in Fig. 1 which shows a rather large overlap with the areas from which the analytical expressions were drawn. This enables to study and compare the dependence, for all the models considered here including the full EHD solution, of $w$ and $u_{\mathrm{e}}$ on film thickness for both configurations, circular firstly, and then elliptical.

The last important point to consider for conducting an objective analysis concerns the integration of the lubricant properties. Indeed, the numerical model used in this work includes two physical laws that quantitatively describe the actual response of the lubricant subjected to contact conditions, while the analytical EHD models are based on empirical expressions, like the Barus, Roelands or DowsonHigginson equations. Concerning the viscosity-pressure dependence, it should be reminded that Hamrock \& Dowson [4] were aware of the weakness of the Barus law. In their expressions they preferred to consider $\alpha^{*}$, the reciprocal asymptotic isoviscous pressure as proposed by Blok [21], instead of the classical secant pressure-viscosity coefficient based on an exponential dependence, i.e., on the so-called Barus law. Interestingly, the use of $\alpha^{*}$ some decades later has confirmed [22, 3], by comparison with experiments, that this parameter was really relevant to predict film thickness. Following this agreement, the reciprocal asymptotic isoviscous pressure was used to calculate film thickness from the analytical expressions.

\section{Results and discussion on circular contacts}

Results are expressed as the relative film thickness deviation given by each analytical equation to our numerical model (noted $h_{\text {ref }}$ thereafter), a positive value meaning an overestimation:

$$
\Delta h / h_{\text {ref }}=\left(h_{\text {mod }}-h_{\text {ref }}\right) / h_{\text {ref }}
$$

where $h$ can be either $h_{\mathrm{c}}$ or $h_{\mathrm{m}}$ and $h_{\text {mod }}$ refers to a 
prediction by an analytical model, i.e., Masjedi \& Khonsari [9], Chittenden et al. [8], Evans \& Snidle [7], Hamrock \& Dowson [4], Nijenbanning et al. [5] or Chevalier [6] expressions. Throughout the rest of the paper, results are graphically reported according the following order: Case 1, Case 3, Case 5, Case 2, and Case 4 . This enables first to assess and compare the influence of an increasing normal load $(120,800$, and 2,500 N, respectively Case 1, Case 3 and Case 5), the remaining parameters being kept constant, and then to pursue the analysis to the entrainment speed influence (from 0.5 to $10 \mathrm{~m} / \mathrm{s}$ ), respectively for Case 2 and Case $4, w$ being constant and equal to $800 \mathrm{~N}$.

Figure 2 presents a comparison of the central film thickness results, expressed by $\Delta h_{\mathrm{c}} / h_{\mathrm{c} \text {,ref }}$, given by the analytical film thickness equations mentioned before which are suitable for circular contacts. The 5 models are, in average, rather accurate and capable to estimate $h_{\mathrm{c}}$ with an acceptable precision (represented by a bold dotted line in Fig. 2) of $9 \%$ in average with a standard deviation of $6 \%$ : the interval of confidence (defined by $+/-$ the standard deviation to the mean gap) is delimited by two thin dotted lines in Fig. 2. The results are not uniform across the models: those computed from the equation of Chittenden et al. [8] are in excellent agreement (within 3\%) with the numerical solutions for the 5 reference cases, whereas the models of Evans \& Snidle [7] (in particular at high load and/or low velocity) and of Nijenbanning et al. [5] (in a rather uniform manner) deviate more

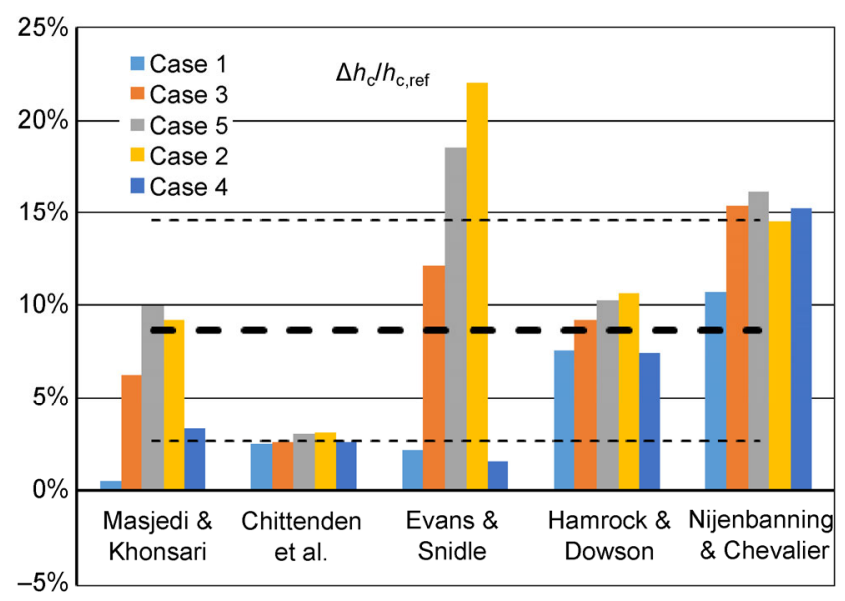

Fig. 2 Relative deviations given by analytical EHD film thickness expressions on $h_{\mathrm{c}}$, the central film thickness, for the 5 circular reference cases defined in Table 1. significantly. A general and clear trend is however revealed, for the conditions simulated in this study: all the analytical EHD equations overestimate the central film thickness, on average by $9 \%$ which can be considered nevertheless as a moderate discrepancy.

The relative deviations on minimum film thickness predictions are reported in Fig. 3, expressed in the same way as in Fig. 2. For $h_{\mathrm{m}}$, the discrepancy is much larger than for $h_{\mathrm{c}}$ and reaches an average value of $37 \%$ for the 5 reference cases of Table 1, with a standard deviation of $34 \%$. Nevertheless, the combined Nijenbanning \& Chevalier model $[3,5]$ provides a rather fair prediction of $h_{\mathrm{m}}$ with a mean overestimation of $+11 \%$, while the use of Evans \& Snidle expression results in the unique, but very low, underestimation for Case 4 , of $-1.2 \%$. The three other analytical equations predict strongly optimistic minimum film thicknesses: they overestimate $h_{\mathrm{m}}$ by nearly $+50 \%$ with several occurrences exceeding $+80 \%$, especially at high load and/or low velocity conditions. These deviations are certainly too large - if not unacceptable - to insure safe working conditions of lubricated mechanisms, given the current technical and environmental demands that lead to lubricate with thinner and thinner lubricating films. Moreover, they could lead to erroneous lubrication regime estimation, the actual minimum film thickness being half-value of the analytically calculated ones.

In summary, the analytical EHD equations generally overestimate film thickness in circular contacts, to a

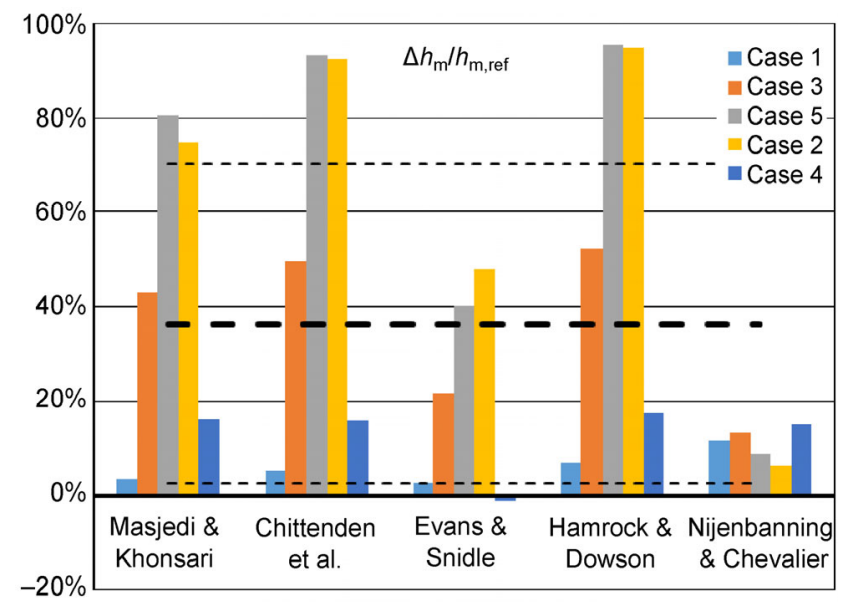

Fig. 3 Relative deviations given by analytical EHD film thickness expressions on $h_{\mathrm{m}}$, the minimum film thickness, for the 5 circular reference cases defined in Table 1. 
much larger extent for $h_{\mathrm{m}}$, the minimum value, while the prediction appears acceptable, for an engineering point of view, for $h_{c}$ the central film thickness. This global discrepancy cannot be, to first order, attributed to the different manners of taking into account the physical properties of the lubricant in the full numerical model. If such were the cases, the agreement on $h_{\mathrm{m}}$ may have been more satisfying, given that minimum film thickness takes place where the pressure approaches its ambient value and therefore where the density and viscosity become closer to the ambient values. However, it is the opposite trend that is observed.

Clearly, the results of this comparison between analytical and numerical methodologies are in line with some previous findings. Concerning central film thickness, van Leeuwen $[1,2]$ concluded that for both moderately-loaded and highly-loaded EHD contacts, Chittenden et al. [8] formula was the more accurate and that its validity transcended the area where it was originally designed for. As for minimum film thickness, the use of Chevalier ratios [6] combined with the Nijenbanning et al. [5] formula has shown, from experimental confrontation, to be the more consistent over very wide ranges of the $(M, L)$ parameters [3]. But perhaps the crucial point to emphasize here lies in the fact that the results of the previous works were based on experimental measurements and are now fully confirmed by the current study which relies on a purely numerical and modeling approach.

\section{Results and discussion on elliptical contacts}

The consideration of elliptical contacts excludes the Evans \& Snidle equations and the Chevalier table, all designed for the circular geometry. Moreover, there are two options to represent ellipticity, the first one based on $k=\frac{b}{a}$ where $a$ is the contact length and $b$ its width, the second relies on $D$, which expresses the ratio of the reduced radii of curvature at the contact center. $k$ was selected here, given that most of the works on elliptical contacts have used this parameter to represent ellipticity.

Figures 4(a) and 4(b) compare, in $M-k / D$ and $k / D-L$ charts, the domains on which the Hamrock \& Dowson
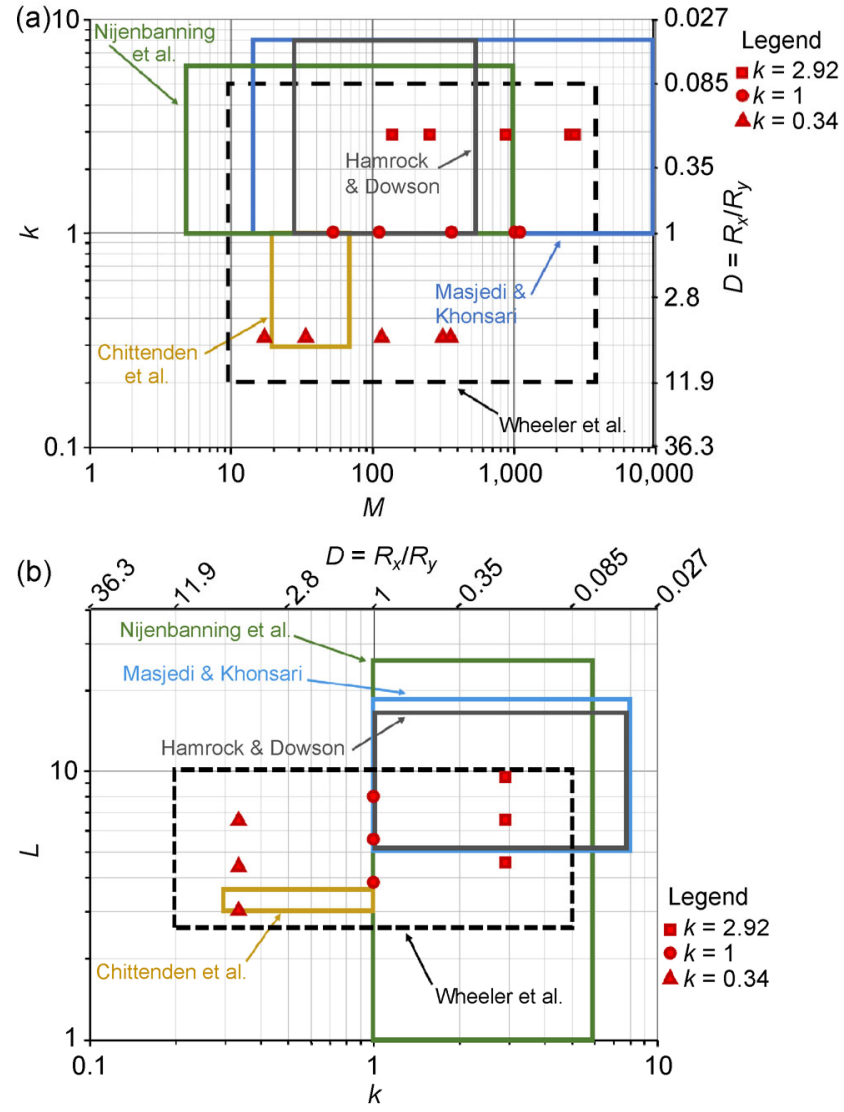

Fig. 4 Domains on which EHD film thickness equations for elliptical contacts were established. The black bold dotted line indicates the region where the full numerical model was applied in Ref. [11]. The square and triangle show the ranges on which the comparison was conducted for two elliptical configurations defined by $k=2.92$ and 0.34 (or $D=0.2$ and 5), respectively: (a) expressed in $M-k / D$ chart, and (b) expressed in $k / D-L$ chart.

[4], Nijenbanning et al. [5], Chittenden et al. [8] and Masjedi \& Khonsari [9] were derived for elliptical contacts. Note that Chittenden and his co-authors were the only ones to explore the case $k<1$ (narrow or slender configuration), and that they included the Hamrock \& Dowson results to establish their analytical models, thus valid for both slender and wide configurations. This is also the case in the paper by Wheeler et al. [11] who explored $k$ values ranging from 0.2 to 5 delimited by the bold black dotted lines in Figs. 4(a) and 4(b).

In the following, the five reference cases of Table 1 will be combined with two configurations representative of wide and slender elliptical contacts, characterized by $k=2.92$ and 0.34 (or $D=0.2$ and 5), respectively. In the numerical model, the initial values of the radiuses 
of curvature along the main axes were varied, keeping all the remaining parameters constant. As a consequence, the ranges of dimensionless parameters have been much extended compared to the circular cases and cover $M \in[131,2,736], L \in[4.5,9.6]$ and $M \in$ $[17,358], L \in[3,6.4]$, respectively. This is also visible in Figs. 4(a) and 4(b) where the values corresponding to the circular configuration (red dots) are exceeded for the elliptical cases in $M$ and $L$, towards lower and higher extrema.

\subsection{Wide elliptical contacts: $k=2.92$}

First note that Chittenden et al. [8], Hamrock \& Dowson [4], and Nijenbanning et al. [5] formulae were extrapolated from the domains in $M$ they were established for reference Case 2 (low speed, medium load, $M=2,476$ ) and Case 5 (medium speed, high load, $M=2,736$ ), see Fig. 4(a). However the results for these particular cases do not show significant differences with those calculated under regular conditions, i.e., without extrapolation, see Fig. 5 for the central film thickness for instance. In some ways, these results demonstrate the relative robustness of the 3 models mentioned just above.

The results are expressed as before for circular contacts, using Eq. (1). Overall, the confrontation between analytical film thickness expressions and the full EHD model for wide elliptical contacts results in similar trends as for the circular case: firstly $h_{\mathrm{c}}$ is systematically overestimated (see Fig. 5) and secondly,

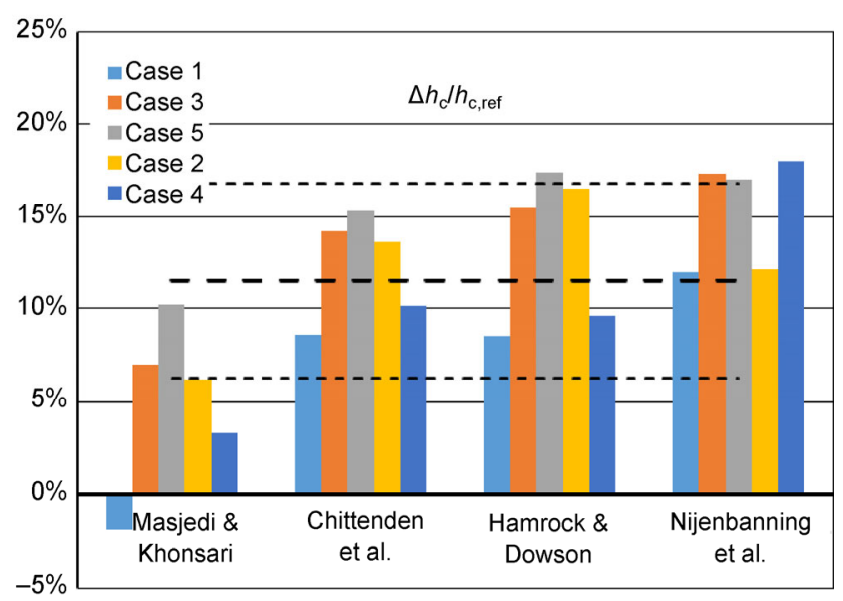

Fig. 5 Relative deviations given by analytical EHD film thickness expressions on $h_{\mathrm{c}}$, the central film thickness, for the 5 wide elliptical reference cases defined by $k=2.92$. a mean discrepancy of $+12 \%$ is found, with a standard deviation of $5 \%$. In a logical way, the Masjedi \& Khonsari model [9] proves to be the most accurate in predicting central thickness (within 5\%) in wide elliptical contacts $(k=2.92$ or $D=0.2)$. This is the most recent model (published in 2015), thus one can reasonably expect a fairer prediction compared with earlier models. Moreover, it has been established over the widest area in $M[5,10,000]$ and for $k$ values ranging from 1 up to 8 : it was thus applied within its domain of validity and any extrapolation was introduced which might have resulted in some further deviation.

When it comes to $h_{\mathrm{m}}$ the same remark as for the central film thickness applies on the domains of validity of the analytical expressions, but here it was not possible to extrapolate the Nijenbanning et al. model because the tabulated ratio $h_{\mathrm{c}} / h_{\mathrm{m}}$ at $M>1,000$ has not been quantified in Ref. [5]. Thus results for Case 2 and Case 5 are missing for this expression. The minimum film thickness results for $k=2.92$ are plotted in Fig. 6.

The minimum film thickness predictions by the analytical equations always lead to overestimation, and appear of a satisfying precision: the mean discrepancy is equal to $+6 \%$, and the standard deviation of the same value. The Nijenbanning et al. [5] table gives the more accurate estimate of $h_{\mathrm{m}}$ but is limited up to $M=1,000$, thus to Cases 1,3 and 4 only in this study. Compared to the circular case, the fact that the

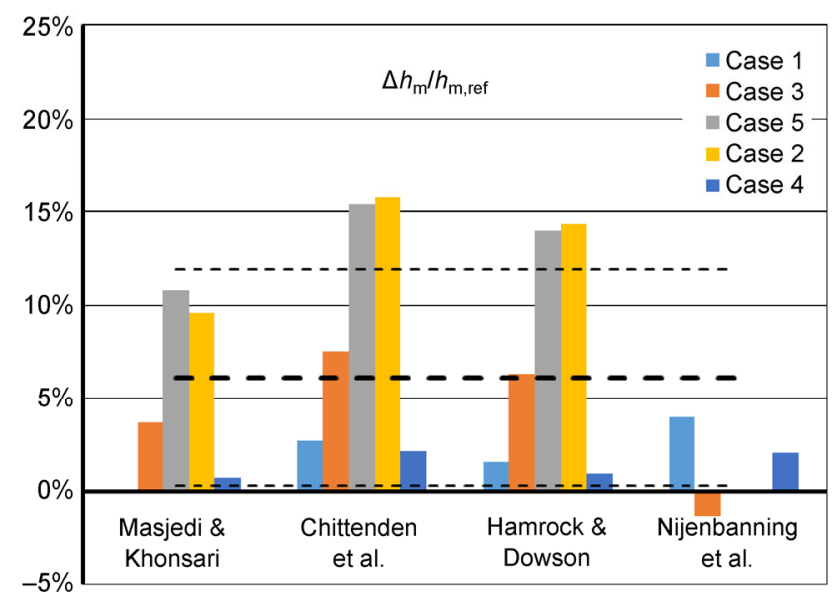

Fig. 6 Relative deviations given by analytical EHD film thickness expressions on $h_{\mathrm{m}}$, the minimum film thickness, for 5 wide elliptical reference cases defined by $k=2.92$. 
analytical models appear more accurate, or less false in the perspective of a quantitative approach, in the elliptical configuration may seem surprising. However, in this section film thicknesses have been computed for $k=2.92$ which still denotes a rather marked wide elliptical configuration. Under these circumstances, hydrodynamic effects are largely dominated by Poiseuille flows in the entrainment speed direction, the contact approaches the infinitely wide case and the places where the minimum film thickness occurs deviate from the lateral lobes towards the contact exit area [11]. For instance, with $k=2.92$ and $W=800 \mathrm{~N}$ (the medium load case) one reaches the equality between the classical minimum thicknesses found on the lobes and the film thickness at the center of the exit zone of the contact [11], where the minima would occur if $k$ was increased further, as in the case of line contacts. Here, for $k=2.92$ the average $h_{\mathrm{c}} / h_{\mathrm{m}}$ ratio obtained from all models (analytical and numerical) is equal to $1.28(+28 \%)$, against $2.5(+150 \%)$ in the circular configuration. The $h_{\mathrm{c}}$ and $h_{\mathrm{m}}$ values becoming closer, there is no reason why their prediction would give very different trends, in terms of accuracy.

\subsection{Slender elliptical contacts: $k=0.34$}

In this configuration, the only available analytical model is that of Chittenden et al. [8]. However, even if the authors have specifically explored $k \in[0.3,1]$, its range of application in terms of $(M, L)$ domain was defined for $M \in[20,70]$ and $L \in[3,3.5]$ (see Fig. 1), which is rather limited compared with the domain explored here ( $M \in[17,358], L \in[3,6.4])$, see Figs. 4(a) and 4(b). This model was thus significantly extrapolated for most cases to obtain the results reported in Fig. 7.

For the first time in this work, central film thickness is underestimated (see Fig. 7 left) with a mean relative gap of $-20 \%$ and a standard deviation of $9 \%$ : these values are rather similar to those reported concerning $h_{\mathrm{c}}$ prediction in the circular and wide elliptical cases. In contrast, the minimum thickness is dramatically overestimated with an average relative difference of the order of $140 \%$. This tendency clearly shows that the Chittenden et al. [8] model has no capability to properly capture the underlying mechanisms occurring in slender elliptical contacts when extrapolated to rather high $M$ values. Two main phenomena intervene in such conditions. The hydrodynamics effects are dominated by the lateral Poiseuille flow rates along the directions transverse to the entrainment velocity [11]. In the meantime a relatively larger radius of curvature in the $u_{\mathrm{e}}$ direction reduces the wedge effect and thus the film building ability. The two effects are cumulative to generate a dramatic film thickness reduction especially on $h_{\mathrm{m}}$, which leads to unusual $h_{\mathrm{c}} / h_{\mathrm{m}}$ ratios. For the 5 reference cases considered here, this ratio is equal to 6.2 in average, which is a much larger value than in the circular or wide configurations. Furthermore, it can take values close to or higher than 9, as for the reference Case 2 and Case 5 where the minimum film thickness calculated from the full
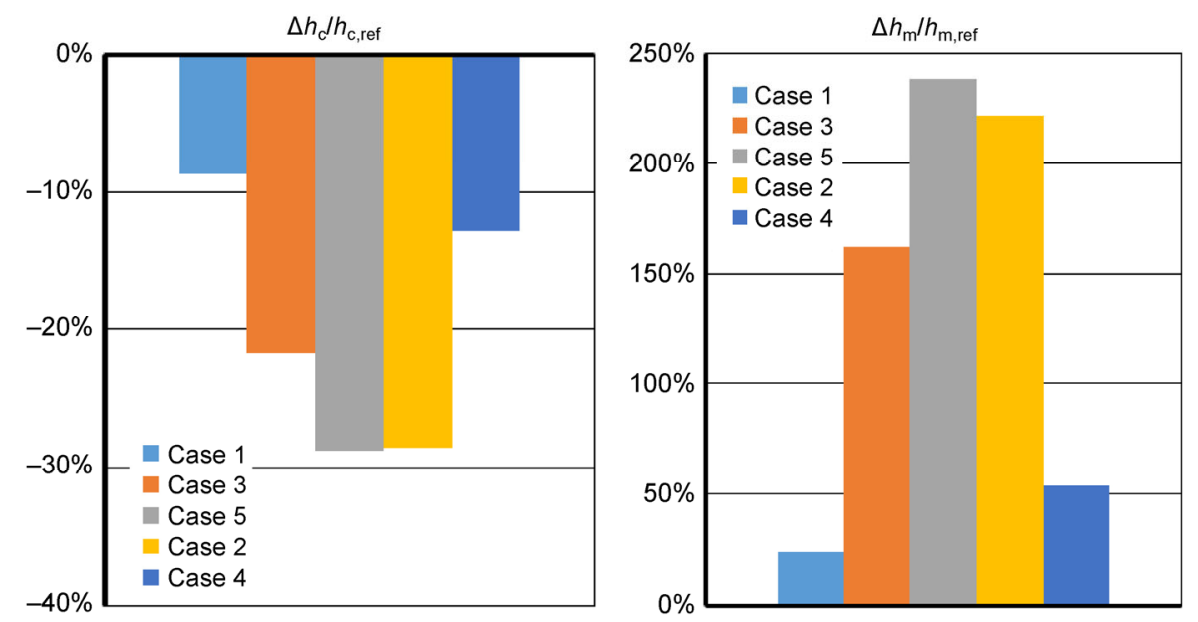

Fig. 7 Relative deviations given by the Chittenden et al. [8] analytical formulae on $h_{\mathrm{c}}$ (left) and $h_{\mathrm{m}}$ (right) for the 5 elliptical reference cases in a slender configuration defined by $k=0.34$. 
EHD model drops down to 13 and $28 \mathrm{~nm}$, respectively. This underlines the impossibility, for the slender configuration, to extrapolate the Chittenden et al. [8] model to $M$ values outside the range the expression was designed for.

\section{Conclusions}

Thin film lubrication and EHL are rather young domains of tribology and of science and technology in general, which really emerged about 70 years ago. One could have think, with the tremendous development of experimental techniques and computational tools, that they could become mature and well understood after this period. That was somehow one of the very first objectives of this work to ensure that one is able to predict analytically the lubricant film thickness in point contacts operated under the simplest conditions (Newtonian fluid, smooth surfaces, and no thermal effect).

Based upon a selection of well-known and widely used semi-analytical expressions, the first step consisted to present and compare their domains of validity, expressed through the $M, L$ and $k$ (or $D$ ) parameters. The differences among the models and the ranges not covered - or covered by only some of the formulaehave been identified and highlighted, especially when extrapolations were required to be carry out.

The comparison between the analytical predictions and the results from a full EHD solver has been then examined for circular contacts. In spite of being the most studied configuration from the earlier stages of development of thin film lubrication and EHL, this first assessment showed that film thickness was systematically overevaluated: the central film thickness was rather accurately predicted whereas a much larger discrepancy was obtained on the minimum film thickness.

The extension to elliptical cases, both slender and wide, was conducted with a more limited number of analytical models. The comparison was found to be more favorable in the case of wide elliptical contacts: film thick thickness was still over estimated but in a lower extent, and especially for the minimum film thickness where the best agreement between analytical and numerical predictions was obtained. In contrast, the worst situation was pointed out in the case of slender contacts, for which only one analytical model was studied and showed its quasi inability for extrapolation to larger $M$ values than those it was derived for.

Whatever the geometrical configuration, circular or elliptical, it is clear that the reference Case 5 and Case 2 , namely the highly loaded and low velocity cases, gathered the largest discrepancies with the analytical models. This is certainly a major weakness because such cases correspond in fact to conditions more and more frequently found nowadays in lubricated systems: very thin lubricating films in line with the unceasing drop of film thickness with time, and heavily loaded contacts as those found for instance in rolling element bearings or in gears.

From the results of the current work, it is clear that analytical models can, at best, provide a qualitative estimate of film thickness. In such an approach, it could be recommended to use the Chittenden et al. [8] equation for estimating $h_{\mathrm{c}}$ and the Nijenbanning et al. expression [5] combined with the Chevalier table [6, 3] for predicting $h_{\mathrm{m}}$ in circular contacts, the Masjedi \& Khonsari models [9] for $h_{\mathrm{c}}$ and $h_{\mathrm{m}}$ in wide elliptical contacts. The question of the slender elliptical contacts remains open, pending a suitable analytical model. A great care should be taken for establishing the lubrication regime: all the analytical models investigated in this work over predict minimum film thickness, which may lead to estimate erroneous frontiers between full film and mixed lubrication regimes.

Given the findings of this work and the conclusions and recommendations reported above, the most reliable approach to predict film thickness in EHD point contacts should, in authors' opinion, rely over a full numerical model. It is quantitative by nature and can include the actual lubricant behavior-obtained independently from tribological tests-and various other features not accounted for here.

\section{Acknowledgment}

This work was partly financed by SKF in the framework of the global program "Advanced Bearing Lubrication". 


\section{Appendix: Analytical expressions}

\section{Hamrock \& Dowson [4]:}

$h_{\mathrm{c}} / R_{x}=2.69 U^{0.67} G^{0.53} W^{-0.067}\left(1-0.61 e^{-0.75\left(R_{y} / R_{x}\right)^{0.64}}\right)$

$h_{\mathrm{m}} / R_{x}=3.63 U^{0.68} G^{0.49} W^{-0.073}\left(1-e^{-0.70\left(R_{y} / R_{x}\right)^{0.64}}\right)$

\section{Evans \& Snidle [7]:}

$h_{\mathrm{c}} /\left(R_{x}(2 U)^{0.5}\right)=1.7 M^{-0.026} L^{0.40}$

$h_{\mathrm{m}} /\left(R_{x}(2 U)^{0.5}\right)=1.9 M^{-0.17} L^{0.34}$

\section{Chittenden et al. [8]:}

$h_{\mathrm{c}} / R_{x}=4.31 U^{0.68} G^{0.49} W^{-0.073}\left(1-e^{-1.23\left(R_{y} / R_{x}\right)^{2 / 3}}\right)$

$h_{\mathrm{m}} / R_{x}=3.68 U^{0.68} G^{0.49} W^{-0.073}\left(1-e^{-0.67\left(R_{y} / R_{x}\right)^{2 / 3}}\right)$

\section{Nijenbanning et al. [5]:}

$$
\begin{aligned}
h_{\mathrm{c}} /\left(R_{x}(2 U)^{0.5}\right)= & \left(\left(H_{\mathrm{RI}}^{3 / 2}+\left(H_{\mathrm{EI}}^{-4}+H_{00}^{-4}\right)^{-3 / 8}\right)^{2 s / 3}\right. \\
& \left.+\left(H_{\mathrm{RP}}^{-8}+H_{\mathrm{EP}}^{-8}\right)^{-s / 8}\right)^{1 / s}
\end{aligned}
$$

with:

$$
\begin{aligned}
& D=R_{x} / R_{y} \\
& S=1.5\left(1+e^{-1.2 / H_{\mathrm{EI}} / H_{\mathrm{RI}}}\right) \\
& H_{00}=1.8 D^{-1} \\
& H_{\mathrm{RI}}=145\left(1+0.796 D^{14 / 15}\right)^{-15 / 7} D^{-1} M^{-2} \\
& H_{\mathrm{EI}}=3.18\left(1+0.006 \ln (D)+0.63 D^{4 / 7}\right)^{-14 / 15} D^{-1 / 15} M^{-2 / 15} \\
& H_{\mathrm{RP}}=1.29(1+0.691 D)^{-2 / 3} L^{2 / 3} \\
& H_{\mathrm{EP}}=1.48\left(1+0.006 \ln (D)+0.63 D^{4 / 7}\right)^{-7 / 20} D^{-1 / 24} M^{-1 / 12} L^{3 / 4}
\end{aligned}
$$

\begin{tabular}{|c|c|c|c|c|c|c|c|c|c|}
\hline \multirow{2}{*}{\multicolumn{2}{|c|}{$D=0.4$}} & \multicolumn{8}{|c|}{$M$} \\
\hline & & 5 & 10 & 20 & 50 & 100 & 200 & 500 & 1000 \\
\hline \multirow{6}{*}{$L$} & 0 & 1.3 & 1.3 & 1.3 & 1.3 & 1.3 & 1.3 & 1.3 & 1.3 \\
\hline & 1 & 1.3 & 1.3 & 1.3 & 1.3 & 1.4 & 1.4 & 1.5 & 1.6 \\
\hline & 2.5 & 1.3 & 1.3 & 1.3 & 1.4 & 1.5 & 1.6 & 1.7 & 1.8 \\
\hline & 5 & 1.3 & 1.3 & 1.4 & 1.5 & 1.5 & 1.6 & 1.8 & 2 \\
\hline & 10 & 1.3 & 1.4 & 1.4 & 1.5 & 1.5 & 1.6 & 1.8 & 2 \\
\hline & 25 & - & 1.4 & 1.3 & 1.5 & 1.6 & 1.6 & 1.7 & 1.9 \\
\hline \multirow{2}{*}{\multicolumn{2}{|c|}{$D=0.2$}} & \multicolumn{8}{|c|}{$\bar{M}$} \\
\hline & & 5 & 10 & 20 & 50 & 100 & 200 & 500 & 1000 \\
\hline \multirow{6}{*}{$L$} & 0 & 1.2 & 1.3 & 1.3 & 1.3 & 1.3 & 1.3 & 1.3 & 1.3 \\
\hline & 1 & 1.2 & 1.3 & 1.3 & 1.3 & 1.3 & 1.3 & 1.3 & 1.4 \\
\hline & 2.5 & 1.2 & 1.3 & 1.3 & 1.3 & 1.3 & 1.3 & 1.3 & 1.4 \\
\hline & 5 & 1.2 & 1.3 & 1.3 & 1.3 & 1.3 & 1.4 & 1.4 & 1.5 \\
\hline & 10 & 1.2 & 1.3 & 1.3 & 1.4 & 1.4 & 1.4 & 1.5 & 1.5 \\
\hline & 25 & - & 1.3 & 1.3 & 1.4 & 1.4 & 1.4 & 1.5 & 1.5 \\
\hline
\end{tabular}

$h_{\mathrm{m}}$ is obtained from the $h_{\mathrm{c}} / h_{\mathrm{m}}$ ratios reported in the following tables:

\begin{tabular}{c|c|c|c|c|c|c|c|c}
\hline \multirow{2}{*}{$\begin{array}{c}D=1 \text { from } \\
\text { Chevalier } \\
{[3,6]}\end{array}$} & \multicolumn{8}{c}{$M$} \\
\cline { 2 - 9 } \multicolumn{1}{c}{} & 1 & 3 & 10 & 30 & 100 & 300 & 1000 \\
\hline \multirow{4}{*}{$L$} & 0 & 1.2645 & 1.2635 & 1.26 & 1.25 & 1.33 & 1.48 & 1.93 \\
\cline { 2 - 9 } & 2 & 1.2915 & 1.3045 & 1.35 & 1.48 & 1.8 & 2.23 & 3.28 \\
\cline { 2 - 9 } & 5 & 1.251 & 1.273 & 1.35 & 1.57 & 1.92 & 2.42 & 3.43 \\
\cline { 2 - 9 } & 10 & 1.2645 & 1.2835 & 1.35 & 1.54 & 1.87 & 2.33 & 3.2 \\
\cline { 2 - 9 } & 20 & 1.2425 & 1.2575 & 1.31 & 1.46 & 1.72 & 2.08 & 2.79 \\
\cline { 2 - 9 } & 40 & 1.1985 & 1.2055 & 1.23 & 1.3 & 1.42 & 1.58 & 1.97 \\
\cline { 2 - 9 } & 60 & 1.1545 & 1.1535 & 1.15 & 1.14 & 1.12 & 1.08 & 1.15 \\
\hline
\end{tabular}

\section{Masjedi \& Khonsari [9]:}

$$
\begin{aligned}
& h_{\mathrm{c}} / R_{x}=3.672 U^{0.663 k^{0.025}} G^{0.502 k^{0.064}} W^{-0.045 k^{0.18}}\left(1-0.573 e^{-0.74 k}\right) \\
& h_{\mathrm{m}} / R_{x}=1.637 U^{0.711 k^{-0.023}} G^{0.650 k^{-0.045}} W^{-0.09 k^{-0.15}}\left(1-0.974 e^{-0.676 k}\right)
\end{aligned}
$$

Open Access: The articles published in this journal are distributed under the terms of the Creative Commons Attribution 4.0 International License (http:// creativecommons.org/licenses/by/4.0/), which permits unrestricted use, distribution, and reproduction in any medium, provided you give appropriate credit to the original author(s) and the source, provide a link to the Creative Commons license, and indicate if changes were made.

\section{References}

[1] van Leeuwen $H$. The determination of the pressureviscosity coefficient of a lubricant through an accurate film thickness formula and accurate film thickness measurements. Proc IMechE, Part J: J Eng Tribol 223(8): 1143-1163 (2009)

[2] van Leeuwen H. The determination of the pressureviscosity coefficient of a lubricant through an accurate film thickness formula and accurate film thickness measurements. Part 2: High L values. Proc IMechE, Part J: J Eng Tribol 225(6): 449-464 (2011)

[3] Chaomleffel J P, Dalmaz G, Vergne P. Experimental results and analytical predictions of EHL film thickness. Tribol Int 40(10-12): 1543-1552 (2007) 
[4] Hamrock B J, Dowson D. Isothermal elastohydrodynamic lubrication of point contacts Part III - Fully flooded results. Trans ASME J Lubr Technol 99(2): 264-276 (1977)

[5] Nijenbanning G, Venner C H, Moes H. Film thickness in elastohydrodynamically lubricated elliptic contacts. Wear 176: 217-229 (1994)

[6] Chevalier F. Modélisation des conditions d'alimentation dans les contacts élastohydrodynamiques ponctuels. (in French). PhD thesis, INSA de Lyon, France, 1996.

[7] Evans P, Snidle R. The isothermal elastohydrodynamic lubrication of spheres. ASME J Lubr Technol 103: 547-557 (1981)

[8] Chittenden R J, Dowson D, Dunn J F, Taylor C M. A theoretical analysis of the isothermal elastohydrodynamic lubrication of concentrated contacts-Part 2: General case, with lubricant entrainment along either principal axis of the hertzian contact ellipse or at some intermediate angle. Proc $R$ Soc London A397: 271-294 (1985)

[9] Masjedi M, Khonsari M M. On the effect of surface roughness in point-contact EHL: Formulas for film thickness and asperity load. Tribol Int 82(A): 228-244 (2015)

[10] Moes H. Communication. In Proceedings of the Symposium on Elastohydrodynamic Lubrication, London, 1965: 244-245.

[11] Wheeler J D, Fillot N, Philippon D, Vergne P, MoralesEspejel G E. On the crucial role of ellipticity on ehd film thickness and friction. Proc IMechE, Part J: J Eng Tribol 230(12): 1503-1515 (2016)

[12] Doki-Thonon T, Fillot N, Vergne P, Morales-Espejel G E. Numerical insight into heat transfer and power losses in spinning EHL non-Newtonian point contacts. Proc IMechE Part J: J Eng Tribol 226(1):23-35 (2012)

[13] Doki-Thonon T, Fillot N, Morales Espejel G E, Querry M, Philippon D, Devaux N, Vergne P. A dual experimental /

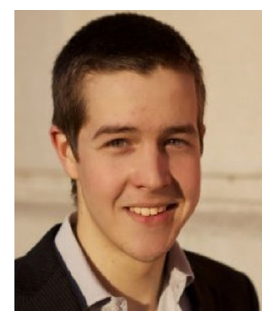

Jean-David WHEELER. He received his M.S. from the Université de Lyon and his Mechanical Engineer degree from the INSA de Lyon in 2013. Since then, he has joined the Laboratoire de Mécanique des

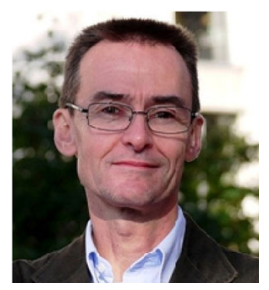

Philippe VERGNE. He is currently CNRS senior researcher at LaMCoS INSA-Lyon. He is the academic leader of the research chair "Lubricated Interfaces for the Future" funded by the SKF company. He obtained a $\mathrm{PhD}$ in mechanical engineering and a Doctor of numerical approach for film thickness analysis in TEHL spinning skewing circular contacts. Tribol Lett 50(1): 115126 (2013)

[14] Habchi W, Demirci I, Eyheramendy D, Morales-Espejel G E, Vergne P. A finite element approach of thin film lubrication in circular EHD contacts. Tribol Int 40(10-12): 1466-1473 (2007)

[15] Habchi W, Eyheramendy D, Vergne P, Morales-Espejel G E. Stabilized fully-coupled finite elements for elastohydrodynamic lubrication problems. Adv Eng Softw 46(1): 4-18 (2012)

[16] Bair S, Mary C, Bouscharain N, Vergne P. An improved Yasutomi correlation for viscosity at high pressure. Proc. IMechE, Part J: J Eng Tribol 227(9): 1056-1060 (2013)

[17] Murnaghan F D. The compressibility of media under extreme pressures. Proc Nat Acad Sci 30(9): 244-247 (1944)

[18] Habchi W, Vergne P, Eyheramendy D, Morales-Espejel G E. Numerical investigation of the use of machinery low viscosity working fluids as lubricants in EHL point contacts. Proc IMechE, Part J: J Eng Tribol 225(6): 465-477 (2011)

[19] Habchi W, Matta C, Joly-Pottuz L, De Barros M, Martin J M, Vergne P. Full film, boundary lubrication and tribochemistry in steel circular contacts lubricated with glycerol. Tribol Lett 42(3): 351-358 (2011)

[20] Wheeler J D. Non-elliptical point contacts: The torus-onplane conjunction. $\mathrm{PhD}$ thesis, INSA Lyon, 2016.

[21] Blok H. Inverse problems in hydrodynamic lubrication and design directives for lubricated flexible surfaces. In Proceedings of the International Symposium on Lubrication and wear, Houston, 1963: 1-151.

[22] Bair S. An experimental verification of the significance of the reciprocal asymptotic isoviscous pressure for EHD lubricants. Tribol Trans 36(2): 153-162 (1993)

Contacts et des Structure (LaMCoS) and has been involved in tribology research, on the topic of EHL. In the frame of the SKF program "Advanced Bearing Lubrication", his focus is the lubrication of the flange roller-end contact. His investigations are led by both experimental and numerical means.

Science (Habilitation) from INSA-Lyon and University of Lyon, in 1985 and 2002 respectively.

His research focuses on the rheological \& tribological behavior of lubricants, on the development of numerical models in the context of EHL \& nano scale lubrication, and of in situ techniques to analyze in details highly confined thin films. 